\title{
INEQUALITIES OF A. MARKOFF AND S. BERNSTEIN FOR POLYNOMIALS AND RELATED FUNCTIONS ${ }^{1}$
}

\author{
A. C. SCHAEFFER
}

Introduction. Weierstrass was the first to prove that an arbitrary continuous function which is defined over a closed finite interval may be uniformly approximated by a sequence of polynomials. The more difficult problem of best approximation by polynomials had earlier been initiated by Tchebycheff. A number of years later, in the early part of the present century, de la Vallée Poussin raised the following question of best approximation: Is it possible to approximate every polygonal line by polynomials of degree $n$ with an error of $o(1 / n)$ as $n$ becomes large? (He had proved that the approximation can be carried out with an error of $O(1 / n)$. This question was answered in the negative by Serge Bernstein in a prize-winning essay on problems of best approximation. In this paper Bernstein proved and made considerable use of an inequality concerning the derivatives of polynomials. This inequality and a related (and earlier) one by Andrew Markoff have been the starting point of a considerable literature. It has been found for example that the underlying ideas of these two inequalities are applicable to a much wider class of functions than polynomials. These inequalities have supplied one approach to questions concerning the derivatives of quasi-analytic functions. A generalization of Bernstein's theorem has been applied to almost periodic functions.

In discussing a mathematical theory we may emphasize either its applications or the salient points of the theory itself. The applications of Bernstein's inequality to problems of approximation (where it has probably had its greatest success) have been treated in the literature; see for example Dunham Jackson's book in the Colloquium Publications of the American Mathematical Society. On the other hand I am unaware of any recent résumé of the literature which has been suggested by the theorems of Markoff and Bernstein, so I shall discuss some of the investigations which have centered about these theorems.

Rational polynomials. It was the chemist Mendeleieff (author of the periodic table of chemistry) who asked the following question: If the bound of a rational polynomial over a given interval is known, how large may its derivative be in this interval? The maximum possible value of the derivative will of course depend on the degree of the poly-

${ }^{1}$ An address delivered before the Los Angeles meeting of the Society on November 23, 1940, by invitation of the Program Committee. 
nomial. Actually Mendeleieff was interested in only the special case in which the polynomial is of degree two, and in this case the question is easily answered. The question for a polynomial of arbitrary degree is more difficult, but was answered in 1889 by A. Markoff [23] who proved the following theorem:

THEOREM I. If $f(x)=\sum_{0}^{n} c_{\nu} x^{\nu}$ is a rational polynomial of degree $n$ and $|f(x)| \leqq 1$ in the interval $-1 \leqq x \leqq 1$ then in the same interval

$$
\left|f^{\prime}(x)\right| \leqq n^{2} \text {. }
$$

The inequality of S. Bernstein [2] which appeared in his paper previously mentioned is as follows:

THEOREM II. Under the conditions of Theorem I

$$
\left|f^{\prime}(x)\right| \leqq n\left(1-x^{2}\right)^{-1 / 2}, \quad-1<x<1 .
$$

Inequalities (1) and (2) are exact in the following sense. The constant $n^{2}$ in (1) cannot be replaced by any lower constant, in fact there is a well known polynomial which satisfies the conditions of Theorem I and whose derivative at the point $x=1$ is equal to $n^{2}$. This polynomial is the $n$th Tchebycheff polynomial

(3) $T_{n}(x)=\cos (n \operatorname{arc} \cos x)=2^{n-1} \prod_{1}^{n}\left\{x-\cos \left(\left(\nu-\frac{1}{2}\right) \pi / n\right)\right\}$.

It satisfies the differential equation

$$
\left(1-x^{2}\right) T_{n}^{\prime \prime}(x)-x T_{n}^{\prime}(x)+n^{2} T_{n}(x)=0 .
$$

However, it is known that at points in the interior of the interval $(-1,1)$ the derivative of $f(x)$ must be strictly less than $n^{2}$. In Bernstein's theorem, on the other hand, the bound for $f^{\prime}(x)$ is given as a function of $x$. This dominant $n\left(1-x^{2}\right)^{-1 / 2}$ is the least possible dominant only at special points in the interval $(-1,1)$. It is, however, asymptotically equal to the precise bound at every fixed point in the interior of the interval as $n$ becomes infinite.

It is easily seen that Bernstein's inequality gives a much lower estimate of $f^{\prime}(x)$ than does Markoff's except for a small neighborhood of the points -1 and +1 . This remark has been used to prove Markoff's theorem with the aid of Bernstein's. If $x$ lies in the interval $|x| \leqq \cos \left(\frac{1}{2} \pi / n\right)$ then (2) shows that

$$
\left|f^{\prime}(x)\right| \leqq \frac{n}{\sin \left(\frac{1}{2} \pi / n\right)} \leqq n^{2} .
$$


Then to prove Markoff's inequality it is only necessary, in view of evident symmetry, to show that (1) is satisfied in the range $\cos \left(\frac{1}{2} \pi / n\right)$ $\leqq x \leqq 1$, and this has been accomplished by several methods. ${ }^{2}$

The above remarks show that if $x$ is a fixed point and $n$ is increasing, the proper bound for $f^{\prime}(x)$ is of order $n$ when $|x|<1$ and of order $n^{2}$ when $|x|=1$. The reason that the order is different in these two cases can be seen from the following result due to Szegö [36]. Let $\Gamma$ represent an open or a closed Jordan curve in the complex $z$-plane, and at any point $z_{0}$ on $\Gamma$ let $\alpha \pi$ be the exterior angle. In the case in which $\Gamma$ is an open curve there will of course be two exterior angles at each point $z_{0}$ except the ends. In this case let $\alpha \pi$ be the larger of the two exterior angles at $z_{0}$. With suitable smoothness conditions on $\Gamma$ we have the following theorem:

TheOREM III. If $f(z)=\sum_{0}^{n} c_{\nu} z^{\nu}$ is a rational polynomial of degree $n$ and $|f(z)| \leqq 1$ on $\Gamma$ then

$$
\left|f^{\prime}\left(z_{0}\right)\right|<c n^{\alpha}, \quad z_{0} \in \Gamma .
$$

Here $c$ is a constant which depends on $z_{0}$ and $\Gamma$, but not on $n$.

It is possible to show that the bound $c n^{\alpha}$ in this inequality is of the precise order as $n$ becomes infinite. In the case in which $\Gamma$ is the interval $(-1,1)$ of the real axis we see that $\alpha=2$ at the endpoints of the interval, so this theorem shows that the derivative is of order $n^{2}$. On the other hand at points in the interior of the interval $\alpha=1$, so at these points the derivative is of order $n$.

Theorem III can be proved by the use of conformal mapping. Let

$$
\omega=\phi(z)=a z+a_{0}+a_{1} / z+\cdots
$$

be a function which maps the exterior of $\Gamma$ onto the exterior of the unit circle in the $\omega$ plane in such a way that the points at infinity correspond. Then the function $f(z) \phi^{-n}(z)$ is regular in the exterior of $\Gamma$, even at infinity. Since it is bounded by 1 on $\Gamma$ it has the same bound outside $\Gamma$,

$$
|f(z)| \leqq|\phi(z)|^{n}
$$

Now the behavior of the mapping function $\phi(z)$ near the boundary of $\Gamma$ has been effectively studied so it is possible to obtain good estimates of $f(z)$ from (4). To obtain an estimate for the derivative of $f(z)$ we write

$$
f^{\prime}\left(z_{0}\right)=\frac{1}{2 \pi i} \int_{C} \frac{f(z)}{\left(z-z_{0}\right)^{2}} d z
$$

${ }^{2}$ See Kellogg [20], M. Riesz [29], Schur [32]. 
where $C$ is a circle with center at $z_{0}$ and properly chosen radius. The theorem is then proved by taking absolute magnitudes under the integral sign and making use of (4).

In the case in which $\Gamma$ is the segment $(-1,1)$ of the real axis the mapping function $\phi(z)$ is especially simple. In this special case Montel [25] had earlier used a similar method to obtain inequalities (1) and (2), although not in their precise form. He showed, for example, that under the conditions of Theorem I the derivative of $f(x)$ is less than $4 n^{2}$.

There are analogous inequalities for the Riemann-Liouville generalized derivatives of positive order $\alpha$ :

$$
D^{\alpha} f(x)=\frac{1}{\Gamma(p-\alpha)} \frac{d^{p}}{d x^{p}} \int_{-1}^{x}(x-t)^{p-\alpha-1} f(t) d t
$$

where $\alpha>0$ and $p$ is the integer part of $\alpha+1$. Montel showed that if $f(x)$ satisfies the conditions of Theorem I then in every interval $\left(-x_{0}, x_{0}\right), 0<x_{0}<1$,

$$
\left|D^{\alpha} f(x)\right| \leqq K n^{\alpha}, \quad K=K\left(\alpha, x_{0}\right), \quad \alpha>0 .
$$

This result was later generalized by Sewell [34] who obtained inequalties for the fractional derivatives of those polynomials which satisfy the conditions of Theorem III.

Trigonometric polynomials. An extension of Theorem II to trigonometric polynomials was found by Bernstein. By a trigonometric polynomial of degree $n$ we mean an expression of the form

$$
F(\theta)=\sum_{0}^{n}\left(a_{\nu} \cos \nu \theta+b_{\nu} \sin \nu \theta\right) .
$$

If in Theorem II we make the substitution $x=\cos \theta$, then, as is well known, $f(\cos \theta)=\sum_{0}^{n} c_{\nu} \cos ^{\nu} \theta$ may be written as a trigonometric polynomial of degree $n$ in which only the cosine terms appear. Since $d \theta=-\left(1-x^{2}\right)^{-1 / 2} d x$, inequality (2) states that

$$
\left|\frac{d}{d \theta} f(\cos \theta)\right| \leqq n \text {. }
$$

More generally we may state the following proposition:

THeOREM IV. If $F(\theta)$ is a trigonometric polynomial of degree $n$ and $|F(\theta)| \leqq 1$ for real $\theta$, then

$$
\left|F^{\prime}(\theta)\right| \leqq n, \quad \theta \text { real. }
$$


This result was proved by Bernstein [2] at the same time as Theorem II, except that in (6) he had $2 n$ in place of $n$. Inequality (6) in the present form first appeared in print in a paper of Fekete [15] who attributes the proof to Fejér. Bernstein [5, p. 39] attributes the proof to Landau. This theorem has a wide application to problems of approximation by trigonometric polynomials. The example $F(\theta) \equiv \sin n \theta$ shows that no lower constant than $n$ will suffice in (6).

As already mentioned, under the substitution $x=\cos \theta$ a rational polynomial is transformed into a cosine polynomial in $\theta$ of the same degree; and the converse is also true. On the other hand, a sine polynomial $F(\theta)=\sum_{1}^{n} b_{\nu} \sin \nu \theta$ is transformed into a function of the form $\left(1-x^{2}\right)^{1 / 2} P_{n-1}(x)$ where $P_{n-1}(x)$ is a rational polynomial of degree $n-1$ in $x$. In this sense the trigonometric polynomials represent a wider class of functions than the rational polynomials. This distinction is of some importance because the class of trigonometric polynomials possesses a flexibility which makes several theorems easier to prove in terms of trigonometric polynomials than in terms of rational polynomials.

Bernstein's proof of Theorem IV was based on a variational method. Simpler proofs of this theorem have been obtained by M. Riesz, F. Riesz, and de la Vallée Poussin. Each of these methods has led to interesting extensions of Theorem IV, so we shall discuss them briefly. M. Riesz' proof is based on an important interpolation formula which expresses the derivative of a trigonometric polynomial in terms of the values of the polynomial at $2 n$ equidistant points.

M. RIESZ' INTERPOLATION FORMULA [29]. If $F(\theta)$ is a trigonometric polynomial of degree $n$ then

$$
F^{\prime}(\theta)=\sum_{0}^{2 n-1}(-1)^{v} \rho_{\nu} F\left(\theta+\theta_{\nu}\right) .
$$

Here $\rho_{\nu}$ and $\theta_{\nu}$ are constants which depend only on $n$ and satisfy $\rho_{\nu}>0$, $\sum \rho_{\nu}=n, 0<\theta_{0}<\theta_{1}<\cdots<\theta_{2 n-1}<2 \pi$.

Theorem IV is an obvious consequence of (7). This interpolation formula was used by Zygmund to obtain a generalization of Theorem IV. Zygmund [42] proved the following:

TheOREM V. Let $p \geqq 1$. If $F(\theta)$ is a trigonometric polynomial of degree $n$ then

$$
\left\{(2 \pi)^{-1} \int_{-\pi}^{\pi}\left|F^{\prime}(\theta)\right| p d \theta\right\}^{1 / p} \leqq n\left\{(2 \pi)^{-1} \int_{-\pi}^{\pi}|F(\theta)| p d \theta\right\}^{1 / p} .
$$


This result is proved by use of M. Riesz' interpolation formula and Jensen's inequality for convex functions. Theorem IV is obtained from (8) by letting $p$ become infinite.

The proof of Theorem IV due to F. Riesz [28] begins with the representation

$$
F^{\prime}(\theta)=\frac{1}{\pi} \int_{0}^{2 \pi} F(\theta+\phi)\left\{\sum_{1}^{n} \nu \sin \nu \phi\right\} d \phi .
$$

This may be obtained by use of the familiar integral representation of the coefficients $a_{\nu}$ and $b_{\nu}$. The underlying idea in this method is the completion of the kernel, an idea which had earlier been used by Landau and by Fejér in other problems. The kernel in (9) is not positive, but since $\Gamma$ is a trigonometric polynomial of degree $n$ we may add to the kernel terms in $\cos \nu \phi$ and $\sin \nu \phi$ where $\nu$ is greater than $n$, and the value of the integral will not be changed. Adding a suitable trigonometric sum in (9) we obtain

$$
F^{\prime}(\theta)=\frac{1}{\pi} \int_{0}^{2 \pi} F(\theta+\phi)\left\{n+2 \sum_{1}^{n}(n-\nu) \cos \nu \phi\right\} \sin n \phi d \phi .
$$

The kernel in this case is more regular than before, although it is not positive. If we take absolute magnitudes under the integral sign and replace $|F|$ by 1 and $|\sin n \phi|$ by 1 , what is left of the integrand is positive (it is essentially the Fejér kernel). Then we may integrate termwise and we obtain

$$
\left|F^{\prime}(\theta)\right| \leqq 2 n \text {. }
$$

This is less precise than Theorem IV, but F. Riesz showed how the kernel could be completed still further, and Theorem IV could be proved by this method.

de la Vallée Poussin's proof [40] of Theorem IV is based on the well known theorem that a trigonometric polynomial of degree $n$ can have at most $2 n$ real zeros modulo $2 \pi$. If $\lambda$ and $\alpha$ are real, $-1<\lambda<1$, the function

$$
g(\theta)=\cos n \theta-\lambda F(\theta+\alpha)
$$

is positive wherever $\cos n \theta=+1$ and is negative wherever $\cos n \theta=-1$. It therefore vanishes at $2 n$ different points modulo $2 \pi$. It can be shown that if the derivative of $F(\theta)$ is greater than $n$, then $\lambda$ and $\alpha$ can be so chosen that $g(\theta)$ has at least $2 n+1$ zeros modulo $2 \pi$. This is impossible since $g(\theta)$ is a trigonometric polynomial of degree $2 n$.

The method of de la Vallée Poussin applies only to polynomials 
with real coefficients, but this is a restriction which is easily removed. For, if $F(\theta)$ is any polynomial which satisfies the conditions of Theorem IV and $\gamma$ is a complex constant of unit modulus then $\gamma F(\theta)$ will satisfy the conditions of Theorem IV, and so will $\Re\{\gamma F(\theta)\}$. But $\gamma$ can be so chosen that the derivative of $\gamma F(\theta)$ at any preassigned point is real, and then the derivative of $\Re\{\gamma F(\theta)\}$ will have the same modulus as $F^{\prime}(\theta)$ at this point. The inequalities which have been mentioned until now hold whether the polynomials have real or complex coefficients, and this is true in all subsequent cases except where the contrary is stated.

de la Vallee Poussin's method can be used to show that under the conditions of Theorem IV we have the sharper inequality

$$
n^{2}(F(\theta))^{2}+\left(F^{\prime}(\theta)\right)^{2} \leqq n^{2} .
$$

Here $F(\theta)$ is assumed real. In the general case in which the polynomial is complex we cannot say that the sum of the absolute magnitudes of the two terms on the left is less than $n^{2}$. This is shown by the example $F(\theta)=e^{i n \theta}$. Inequality (12) was first explicitly stated by van der Corput and Schaake [9], although it is implicit in an earlier inequality due to Szegö.

Polynomials in several variables. Now any trigonometric polynomial is the boundary function on the unit circle of a harmonic polynomial of the same degree. If $F(\theta)$ is given by (5) then

$$
F(r, \theta)=\sum_{0}^{n} r^{\nu}\left(a_{\nu} \cos \nu \theta+b_{\nu} \sin \nu \theta\right)
$$

is a harmonic polynomial of degree $n$ and $F(1, \theta)=F(\theta)$. This harmonic function may also be expressed as a polynomial of degree $n$ in the rectangular coordinates $x$ and $y$ under the substitution $x=r \cos \theta$, $y=r \sin \theta$; and it satisfies Laplace's equation $F_{x x}+F_{y y}=0$.

Interpreting Theorem IV from the point of view of harmonic functions, it states that if a harmonic polynomial of degree $n$ is bounded by 1 on the unit circle $r=1$ then its tangential derivative on the unit circle is bounded by $n$. Under the same conditions, how large can the derivative be in other directions? Now the gradient of a function is a vector equal in magnitude to the largest directional derivative of the function. This question is therefore answered in the following theorem of Szegö [37].

Theorem VI. If $F(r, \theta)$ is a harmonic polynomial of degree $n$ and $|F(1, \theta)| \leqq 1$ then for $r \leqq 1$

$$
|\operatorname{grad} F(r, \theta)| \leqq n .
$$


To prove this theorem it is sufficient to show that (14) is satisfied on the unit circle, that is, when $r=1$. Also it is sufficient to show that for all real $\alpha$ the expression

$$
\cos \alpha \frac{\partial F}{\partial r}+\sin \alpha \frac{\partial F}{\partial \theta}
$$

is bounded by $n$. From this point the proof parallels the method of F. Riesz. We obtain by a familiar method of Fourier series an integral representation of (15) which is slightly more complicated than (9). The idea then is to complete the kernel.

At about the same time Kellogg investigated problems concerning the derivatives of general, not necessarily harmonic, polynomials in two and more variables. Suppose that $P(x, y)$ is a polynomial of degree $n$ in rectangular variables, and that it is bounded by 1 in the unit circle $x^{2}+y^{2} \leqq 1$. Here of course we cannot apply the maximum principle of harmonic functions so we assume that the polynomial is bounded in the closed interior of the unit circle, not merely on the boundary. The inequalities in this case will of course not be the same as those that are valid for harmonic polynomials. Along any diameter of the unit circle $P(x, y)=P(r \cos \theta, r \sin \theta)$ will be a polynomial of degree $n$ in $r=\left(x^{2}+y^{2}\right)^{1 / 2}$, where we allow $r$ to take negative values. Theorems I and II then show that its derivative with respect to $r$ (the derivative of $P$ in the radial direction) will be dominated by the smaller of the two numbers $n\left(1-r^{2}\right)^{-1 / 2}$ and $n^{2}$. Here we may ask: How large can the derivative be in other directions? Kellogg answered this by showing that the gradient of $P(x, y)$ is bounded by the smaller of the two numbers $n\left(1-x^{2}-y^{2}\right)^{-1 / 2}$ and $n^{2}$. This includes both Theorem I and Theorem II, for if $f(x)$ satisfies the conditions of Theorem I then it may be considered as a polynomial in $x$ and $y$, with the coefficients of terms in $y$ equal to zero. As such it will be bounded by 1 in the unit circle, and Kellogg's result then shows that the derivative of $f(x)$ must satisfy inequalities (1) and (2).

There are analogous inequalities for polynomials in more than two variables. Kellogg [20] proved the following:

THEOREM VII. If $P\left(x_{1}, x_{2}, \cdots, x_{m}\right)$ is a polynomial of degree $n$ which is bounded by 1 in the unit sphere $x_{1}^{2}+x_{2}^{2}+\cdots+x_{m}^{2} \leqq 1$ then in the same sphere the gradient of $P$ is dominated by the smaller of the two numbers $n\left(1-x_{1}^{2}-x_{2}^{2}-\cdots-x_{m}^{2}\right)^{-1 / 2}$ and $n^{2}$.

Using a rotation of axes, this theorem in the case of more than two 
variables can be deduced from the special case in which there are two variables.

Theorem VI also suggests problems concerning the gradient of polynomials in more than two variables. For harmonic polynomials of three variables which are bounded by 1 on the unit sphere the precise bounds of the gradient are known, except, curiously, when the polynomial is of degree two or three [38]. Unlike Theorem VII, the proper bound for the gradient of harmonic polynomials in three dimensions does not seem to be deducible from the corresponding inequality in the two-dimensional case. The proper bound for the gradient in the three-dimensional case is considerably more complicated than (14).

Entire functions. An extension of Theorem IV to a much wider class of functions than polynomials was noted by Bernstein. Clearly a trigonometric polynomial $F(z)=\sum_{0}^{n}\left(a_{\nu} \cos \nu z+b_{\nu} \sin \nu z\right)$ is periodic with period $2 \pi$, and is also an entire function of the complex variable z. It also belongs to the class of entire functions which are of exponential type, that is, those which satisfy, for some positive constant $\rho$,

$$
F(z)=O\left(e^{\rho|z|}\right)
$$

uniformly in every direction as $|z| \rightarrow \infty$. In the case in which $F(z)$ is a trigonometric polynomial, (16) is true with $\rho$ equal to the degree of the polynomial. Bernstein [4] found that the condition in Theorem IV that $F(z)$ is a trigonometric polynomial can be replaced by the milder condition that it is an entire function of exponential type. $\mathrm{He}$ proved the following:

THEOREM VIII. If $F(z), z=x+i y$, is an entire function of exponential type which satisfies

$$
|F(z)|=O\left(e^{\rho|z|}\right), \quad|F(x)| \leqq 1,
$$

then on the real axis

$$
\left|F^{\prime}(x)\right| \leqq \rho .
$$

In this theorem $\rho$ need not be an integer, and the functions which satisfy these conditions are not in general periodic. This theorem is applicable for example to the general Fourier-Stieltjes transform

$$
F(z)=\int_{-\rho}^{\rho} e^{i z t} d \alpha(t)
$$

where $\alpha(t)$ is of bounded variation. 
If $F(z)$ satisfies the conditions of Theorem VIII it can be shown by repeated use of the Phragmén-Lindelöf principle that

$$
|F(x+i y)| \leqq e^{\rho|y|} .
$$

If $x$ is a point on the real axis let $C$ be a circle with radius $1 / \rho$ and center at $x$, and write

$$
F^{\prime}(x)=\frac{1}{2 \pi i} \int_{C} \frac{F(z)}{(z-x)^{2}} d z .
$$

Then taking absolute magnitudes under the integral sign and making use of (19) we obtain

$$
\left|F^{\prime}(x)\right| \leqq e \rho .
$$

This is less precise than inequality (17). Inequality (17) can be proved using a modification of any of the methods outlined for proving Theorem IV.

Boas [7] obtained sharper results than in Theorem VIII, but under the slightly more stringent condition that $F(z)$ is representable in the form (18), and is bounded by 1 on the real axis.

Higher derivatives. The inequalities which have been mentioned until now have concerned the first derivative of the function. In some cases the proper bounds for the higher derivatives can be inferred by an inductive process from the inequalities for the first derivative. This is the case for example in Theorem IV. If $F(\theta)$ satisfies the conditions of that theorem then the function $F^{\prime}(\theta) / n$ is a trigonometric polynomial of degree $n$, and is bounded by 1 . Hence we infer from (6) that $\left|F^{\prime \prime}(\theta)\right| \leqq n^{2}$. In the same way we obtain the best bounds for all higher derivatives of $F(\theta)$. This inductive process does not give the best bounds in all cases, however. If $f(x)$ is a rational polynomial of degree $n$ and is bounded by 1 in $(-1,1)$ then repeated use of Theorem I will show that $\left|f^{\prime}(x)\right| \leqq n^{2}(n-1)^{2}$. It is known that the true bound for $f^{\prime}(x)$ is less than this. It was Wladimir Markoff (brother of A. Markoff) who found the proper bounds for the higher derivatives of $f(x)$ under these conditions. W. Markoff [24] proved the theorem which follows.

THEOREM IX. Under the conditions of Theorem I

$$
\begin{aligned}
&\left|f^{(p)}(x)\right| \leqq \frac{n^{2}\left(n^{2}-1^{2}\right)\left(n^{2}-2^{2}\right) \cdots\left(n^{2}-(p-1)^{2}\right)}{1 \cdot 3 \cdot 5 \cdots(2 p-1)} ; \\
&-1 \leqq x \leqq 1 ; p=1,2, \cdots, n .
\end{aligned}
$$


The right-hand side of this inequality is exactly equal to $T_{n}^{(p)}(1)$, where $T_{n}(x)$ is the $n$th Tchebycheff polynomial (3). Markoff's proof of this result is based on a variational method. For fixed $p$ suppose that $f(x)$ is an extremal polynomial, that is, suppose its $p$ th derivative assumes the maximum possible value under the conditions of Theorem IX. (It is easily shown that this polynomial exists.) Markoff used a variational method to show that $|f(x)|$ must be equal to 1 at either $n$ or $n+1$ different points in the interval $(-1,1)$. In the latter case $\pm f(x)$ is the $n$th Tchebycheff polynomial, whose derivatives are easily shown to satisfy (20). In the former case it is possible to show that $f(x)$ satisfies a differential equation of the form

$$
1-(f(x))^{2}=\frac{\left(1-x^{2}\right)(x-b)(x-c)}{n^{2}(x-a)^{2}}\left(f^{\prime}(x)\right)^{2} .
$$

Here $a, b, c$ are real constants which depend upon one parameter. Markoff was then able to show that the derivatives of this class of polynomials satisfy (20), but the proof is quite difficult.

The differential equation (21) appeared in a paper of Tchebycheff [39] in 1854 , but the polynomial solutions are often named for Zolotareff, who investigated their properties extensively at a later date. Zolotareff [41] showed that this differential equation admits a polynomial solution if and only if the constants $a, b, c$ satisfy certain equations, which involve elliptic integrals. These elliptic integrals were not used, however, by W. Markoff in the proof of Theorem IX.

W. Markoff also investigated a more general problem: If $k_{0}, k_{1}, \cdots, k_{n}$ are given constants and $f(x)=\sum_{0}^{n} c_{\nu} x^{\nu}$ satisfies the conditions of Theorem I, what is the precise bound for the linear form $\sum c_{\nu} k_{\nu}$ ? The best bound will of course depend upon the constants $k_{\nu}$ as well as upon the degree of the polynomial. By suitably choosing the constants $k_{\nu}$ the linear form can be made equal to any derivative of $f(x)$ at any preassigned point. However, this general problem was not carried so far as the special problem concerning derivatives of $f(x)$.

Duffin and Schaeffer found a shorter proof of Theorem IX. In addition they showed that for inequality (20) to be satisfied it is not necessary to assume that the polynomial is bounded over the entire interval $(-1,1)$. They proved $[13]$ the following fact:

TheOREM X. If $f(x)=\sum_{0}^{n} c_{\nu} x^{\nu}$ is a rational polynomial of degree $n$ and $|f(x)| \leqq 1$ at the $n+1$ points where $\left(T_{n}(x)\right)^{2}=1$, then

$$
\begin{aligned}
& \left|f^{(p)}(x)\right| \leqq \frac{n^{2}\left(n^{2}-1^{2}\right) \cdots\left(n^{2}-(p-1)^{2}\right)}{1 \cdot 3 \cdot 5 \cdots(2 p-1)} \\
& -1 \leqq x \leqq 1 ; p=1,2, \cdots, n \text {. }
\end{aligned}
$$


In the case of the first derivative $(p=1)$ this is a generalization of Theorem I, but there is no analogous generalization of Theorem II, at least in the obvious form. It is possible to show by use of an interpolation formula that if $f(x)$ satisfies the conditions of Theorem $\mathrm{X}$ then the inequality $\left|f^{\prime}(x)\right| \leqq n\left(1-x^{2}\right)^{-1 / 2}$ will be satisfied at special points, but this inequality will not be valid throughout the entire interval $(-1,1)$.

Quasi-analytic functions. The theorems of Markoff and Bernstein lead in a natural way to problems concerning the derivatives of a class of functions which are in general not analytic. One of the simplest results of this nature is the following lemma which is useful in the proof of certain Tauberian theorems: If $f(x)$ is two times differentiable for $x>0$, and if as $x \rightarrow \infty$

$$
f(x)=o(1), \quad f^{\prime \prime}(x)=O(1),
$$

then

$$
f^{\prime}(x)=o(1) .
$$

More general problems of this nature have been investigated by Hardy and Littlewood [16], Landau, and others. One problem which presents itself is:

If $f(x)$ is $n$ times differentiable in a given interval I (finite or infinite), and if throughout $I$,

$$
|f(x)| \leqq 1, \quad\left|f^{(n)}(x)\right| \leqq 1,
$$

what is the proper dominant of $f^{(k)}(x)$ over $I, 0<k<n$ ?

When $I$ is a finite interval the question has not been answered except in special cases. Approximate results were obtained by Ore and Gorny. Ore [26] proved, for example, that under the above conditions the first derivative must satisfy

$$
\left|f^{\prime}(x)\right| \leqq \frac{2(n-1)^{2}}{L}\left\{1+\frac{L^{n}}{n !}\right\}
$$

where $L$ is the length of the interval $I$. His proof is based on Taylor's formula and makes use of A. Markoff's theorem. For a finite interval an exact solution was given by Landau in the special case $n=2$. Landau [22] also investigated problems in which the second derivative satisfies a one-sided condition. Suppose $f(x)$ is real and $|f(x)| \leqq 1$, $f^{\prime \prime}(x) \geqq \alpha$ throughout a finite interval $I$, where $\alpha$ is some constant. Of course if $\alpha$ is too large with respect to the length of the interval 
there will be no function which satisfies these conditions. But if $\alpha$ is small enough, in particular if $\alpha \leqq 0$, the conditions are consistent and it is possible to find a dominant for the first derivative of the function. This dominant varies throughout the interval, becoming infinite at the endpoints.

In the case in which $I$ is an infinite interval and $n$ is arbitrary, the precise bounds for $f^{(k)}(x), 0<k<n$, were obtained by Kolmogoroff [21]. The bound for $f^{(k)}(x)$ which was obtained by Kolmorgoroff is always less than $\pi / 2$, but its precise form is fairly complicated, involving the ratio of two infinite series. This result has been applied to questions concerning quasi-analytic functions. Duffin and Schaeffer [12] altered the problem slightly, making stronger hypotheses and obtaining stronger results. They proved: If the real function $f(x)$ is $n$ times differentiable and if for $-\infty<x<\infty$

(a) $(f(x))^{2} \leqq 1$,

(b) $\left(f^{(n-1)}(x)\right)^{2}+\left(f^{(n)}(x)\right)^{2} \leqq 1$,

then inequality (b) is true when $n$ is replaced by any smaller positive integer. It is possible to obtain Theorem VIII from this result or that of Kolmogoroff by a simple argument.

In conclusion, I wish to acknowledge my indebtedness to Dr. Duffin and Professor Szegö to each of whom I owe a great deal. I am also indebted to Professor Uspensky for several historical remarks.

\section{BIBLIOGRAPHY}

1. N. Achyeser, Über einige Funktionen, die in gegebenen Intervallen am wenigsten von Null abweichen, Bulletin de la Société Physico-Mathématique de Kazan, (3), vol. 3 (1928), pp. 1-69.

2. S. Bernstein, Sur l'ordre de la meilleure approximation des fonctions continues par des polynomes de degré donné, Mémoires de l'Académie Royale de Belgique, (2), vol. 4 (1912), pp. 1-103.

3. S. Bernstein, Sur l'inégalité de W. Markoff, Communications de la Société Mathématique de Kharkow, vol. 14.

4. S. Bernstein, Sur une propriêté des fonctions entières, Comptes Rendus de l'Académie des Sciences, Paris, vol. 176 (1923), pp. 1603-1605.

5. S. Bernstein, Leçons sur les Propriétés Extrémales et la Meilleure Approximation des Fonctions Analytiques d'une Variable Réelle, Paris, 1926.

6. S. Bernstein, Sur un théorème de M. Szegö, Prace Matematyczno-Fizyczne, vol. 44 (1937), pp. 9-14.

7. R. P. Boas, Some theorems on Fourier transforms and conjugate trigonometric integrals, Transactions of this Society, vol. 40 (1936), pp. 287-308.

8. R. P. Boas, The derivative of a trigonometric integral, Journal of the London Mathematical Society, vol. 12 (1937), pp. 164-165.

9. J. G. van der Corput and G. Schaake, Ungleichungen für Polynome und trigonometrische Polynome, Compositio Mathematica, vol. 2 (1935), pp. 321-361. Berichtigung zu: Ungleichungen für Polynome und trigonometrische Polynome, Compositio Mathematica, vol. 3 (1936), p. 128. 
10. R. J. Duffin and A. C. Schaeffer, Some inequalities concerning functions of exponential type, this Bulletin, vol. 43 (1937), pp. 554-556.

11. R. J. Duffin and A. C. Schaeffer, On some inequalities of S. Bernstein and W. Markoff for derivatives of polynomials, this Bulletin, vol. 44 (1938), pp. 289-297.

12. R. J. Duffin and A. C. Schaeffer, On the extension of a functional inequality of S. Bernstein to non-analytic functions, this Bulletin, vol. 46 (1940), pp. 356-368.

13. R. J. Duffin and A. C. Schaeffer, A refinement of an inequality of the brothers Markoff, Transactions of this Society, vol. 50 (1941).

14. L. Fejér, Über einen S. Bernstein-schen Satz über die Derivierte eines trigonometrischen Polynoms, und über die Szegösche Verschärfung desselben, Bulletin of the Calcutta Mathematical Society, vol. 20 (1928-1929), pp. 49-54.

15. M. Fekete, Über einen Satz des Herrn Serge Bernstein, Journal für die reine und angewandte Mathematik, vol. 146 (1916), pp. 88-94.

16. G. H. Hardy and J. E. Littlewood, Contributions to the arithmetic theory of series, Proceedings of the London Mathematical Society, (2), vol. 11 (1913), pp. 411478.

17. E. Hille, G. Szegö, and J. D. Tamarkin, On some generalizations of a theorem of A. Markoff, Duke Mathematical Journal, vol. 3 (1937), pp. 729-739.

18. D. Jackson, The Theory of Approximation, American Mathematical Society Colloquium Publications, vol. 11, 1930.

19. D. Jackson, On the applications of Markoff's theorem to problems of approximation in the complex domain, this Bulletin, vol. 37 (1931), pp. 883-890.

20. O. D. Kellogg, On bounded polynomials in several variables, Mathematische Zeitschrift, vol. 27 (1928), pp. 55-64.

21. A. Kolmogoroff, Une généralisation de l'inégalité de M. J. Hadamard entre les bornes supérieures des dérivees successive d'une fonction, Comptes Rendus de l'Académie des Sciences, Paris, vol. 207 (1938), pp. 764-765.

22. E. Landau, Die Ungleichungen für zweimal differentiierbare Funktionen, Danske Videnskabernes Selskab, Copenhagen, 1925.

23. A. Markoff, On a certain problem of D. I. Mendeleieff, Utcheniya Zapiski Imperatorskoi Akademii Nauk (Russia), vol. 62 (1889), pp. 1-24.

24. W. Markoff, Über Polynome, die in einen gegebenen Intervalle möglichst wenig von Null abweichen, Mathematische Annalen, vol. 77 (1916), pp. 213-258.

25. P. Montel, Sur les polynomes d'approximation, Bulletin de la Société Mathématique de France, vol. 46 (1918), pp. 151-192.

26. O. Ore, On functions with bounded derivatives, Transactions of this Society, vol. 43 (1938), pp. 321-326.

27. G. Pólya and G. Szegö, A ufgaben und Lehrsätze aus der Analysis, Berlin, 1925, vol. 2, pp. 90-91.

28. F. Riesz, Sur les polynomes trigonométriques, Comptes Rendus de l'Académie des Sciences, Paris, vol. 158 (1914), pp. 1657-1661.

29. M. Riesz, Eine trigonometrische Interpolationsformel und einige Ungleichungen für Polynome, Jahresbericht der Deutschen Mathematiker-Vereinigung, vol. 23 (1914), pp. 354-368.

30. A. C. Schaeffer and G. Szegö, Polynomials whose real part is bounded on a given curve in the complex plane, American Journal of Mathematics, vol. 42 (1940), pp. 868876.

31. A. C. Schaeffer and G. Szegö, Inequalities for harmonic polynomials in two and three dimensions, Transactions of this Society, vol. 50 (1941). 
32. I. Schur, Über das Maximum des absoluten Betrages eines Polynoms in einem gegebenen Intervall, Mathematische Zeitschrift, vol. 4 (1919), pp. 271-287.

33. W. E. Sewell, On the modulus of the derivative of a polynomial, this Bulletin, vol. 42 (1936), pp. 699-701.

34. W. E. Sewell, Generalized derivatives and approximation by polynomials, Transactions of this Society, vol. 41 (1937), pp. 84-123.

35. W. E. Sewell, On the polynomial derivative constant for an ellipse, American Mathematical Monthly, vol. 44 (1937), pp. 577-578.

36. G. Szegö, Über einen Satz von A. Markoff, Mathematische Zeitschrift, vol. 23 (1925), pp. 46-61.

37. G. Szegö, Über einen Satz des Herrn Serge Bernstein, Schriften der Königsberger Gelehrten Gesellschaft, vol. 5 (1928), pp. 59-70.

38. G. Szegö, On the gradient of solid harmonic polynomials, Transactions of this Society, vol. 47 (1940), pp. 51-65.

39. P. L. Tchebycheff, Théorie des mécanisms connus sous le nom de parallélogrammes, Mémoires de l'Académie des Sciences de St.-Pétersbourg, vol. 7 (1854), pp. 539-568, cf. also Oeuvres, vol. 1.

40. C. de la Vallée Poussin, Sur le maximum du module de la dérivée d'une expression trigonométrique d'ordre et de module bornés, Comptes Rendus de l'Académie des Sciences, Paris, vol. 166 (1918), pp. 843-846.

41. G. Zolotareff, Sur l'application des fonctions elliptiques aux questions de maxima et minima, Bulletin de l'Académia des Sciences de St.-Pétersbourg, (3), vol. 24 (1878), pp. 305-310; cf. also Oeuvres, vol. 1, pp. 369-374 and vol. 2, pp. 130-166 (the last in Russian).

42. A. Zygmund, A remark on conjugate series, Proceedings of the London Mathematical Society, (2), vol. 34 (1932), pp. 392-400.

\section{ADDED IN PROOF}

43. A. Gorny, Contribution a l'étude des fonctions dérivables d'une variable réelle, Acta Mathematica, vol. 71 (1939), pp. 317-358.

44. C. V. L. Smith, The fractional derivative of a Laplace integral, Duke Mathematical Journal, vol. 8 (1941), pp. 47-77.

\section{STANFORD UNIVERSITY}

